\title{
Packet compression of complex modulation formats based on coherent optical superposition
}

\author{
Francesca Parmigiani*, Joseph Kakande, Liam Jones, Periklis Petropoulos, David J. Richardson \\ Optoelectronics Research Centre, University of Southampton, Southampton SO17 1BJ, UK. \\ Tel.: +44 2380 593141, Fax: +44 2380 593149, *email:frp@orc.soton.ac.uk
}

\section{ABSTRACT}

We propose and numerically demonstrate a packet compression scheme based on re-coding of complex modulation formats through the use of a delay line interferometer (DLI) followed by a temporal gate. A variety of modulation formats can be accommodated and high compression factors can be obtained by using multiple DLIs to achieve higher order modulation formats.

Keywords: Advanced modulation formats, delay line interferometer, time gating, optical coherent superposition.

\section{INTRODUCTION}

Next generation communication systems are expected to exploit complex modulation formats to significantly increase the channel capacity of high-speed optical signals [1-3]. Indeed, optical multilevel modulation carries several information bits in a single symbol, allowing for higher spectral efficiencies (SEs) at the expense of more stringent optical signal to noise ratio (OSNR) requirements. In this context, different types of these new modulation formats may be selectively employed depending on network demands and, thus, a means for moving from one format to another might be very beneficial. Format conversion of single-carrier signals has been achieved using a number of techniques mainly based on nonlinear effects $[4,5]$. In a linear approach, two independent and wavelength shifted to a common carrier signals could be coherently superposed in time to increase the bit per symbol and, thus, the overall SE. However, this solution would impose challenging carrier recovery extraction and phase locking requirements on the two signals. While a simpler solution could rely on coherently superposing the same information-carrying symbol with its delayed replica in time, which can be easily achieved using a delay line interferometer (DLI) [6-8], this approach would inherently cause information redundancy.

In this talk we will numerically study a packet compression scheme based on format conversion using passive coherent superposition [6-8], where an electro-optic gating of the format-converted signal has been implemented to eliminate redundancy, thus, leading to a real increase in the number of bits per symbol and temporal compression factors of up to sixteen. Practical limitations of the scheme in terms of transmission distance and laser linewidth requirements are also investigated.

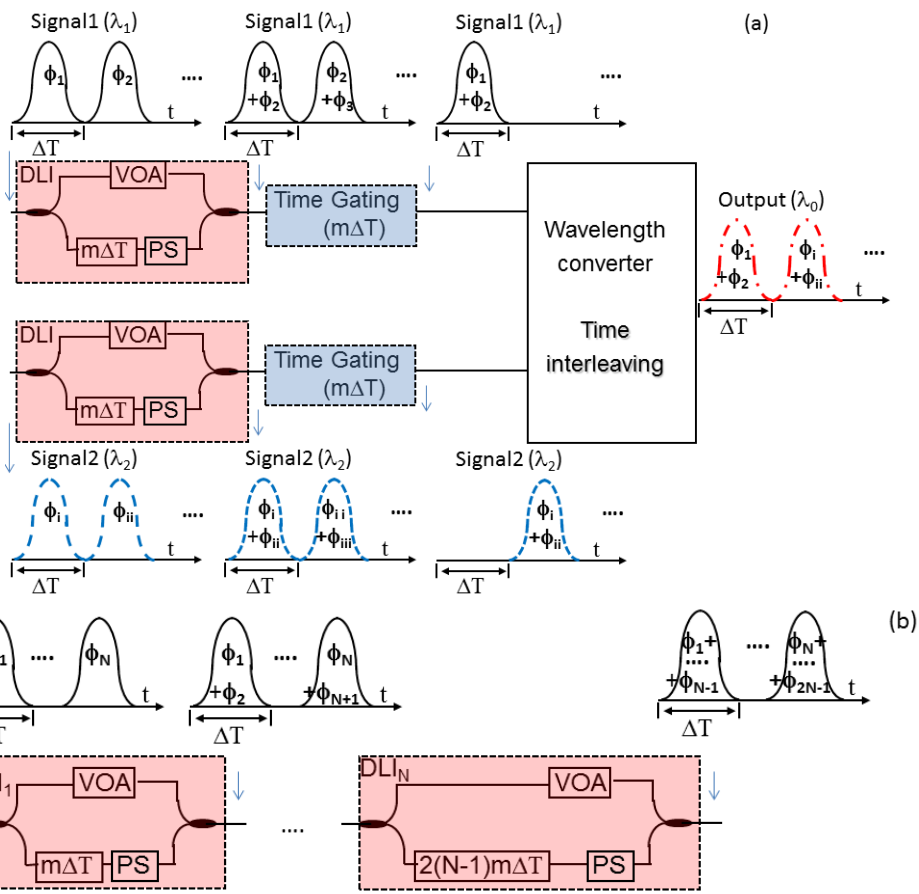

Fig. 1: a) Passive coherent optical superposition operation principle. b) Extension to higher spectral efficiencies, higher compression factors and more complex modulation formats. 


\section{OPERATION PRINCIPLE AND SIMULATED SYSTEM}

The operation principle of the passive coherent optical superposition scheme is shown in Fig.1 (a). A phase and/or amplitude encoded signal at a repetition rate of $1 / \Delta \mathrm{T}$ enters a DLI with free spectral range (FSR) of $\mathrm{m} \Delta \mathrm{T}$, where $\mathrm{m}$ is an integer number and depends on the specific application. For example, in case of packet compression, the value of $\mathrm{m}$ could be chosen in the order of thousands of bits (hundreds of $\mathrm{ns}$ at 10Gbaud). The DLI includes a phase shifter (PS) and a variable optical attenuator (VOA). The signal is split into two copies along the pair of DLI optical paths; the first copy is delayed by $\mathrm{m} \Delta \mathrm{T}$, phase shifted by a pre-determined amount and attenuated depending on the initial/final complex modulation format and coherently added to the second copy at the output of the DLI. Some examples of phase shift and attenuation values are reported in Table 1. Note that to achieve a correct 256 quadrature amplitude modulation (256 QAM), the symbols of the original 16 QAM are not equally spaced in the constellation diagram. If the original signal is different, such as BPSK or quadrature phase shift keying (QPSK), this simply implies that the attenuation value of the DLI to format convert the QPSK signal into the 16QAM one has to be changed from $6 \mathrm{~dB}$ to $12 \mathrm{~dB}$ in Table 1 .

Tab. 1: Phase shift and attenuation values in the DLI for various complex modulation formats.

\begin{tabular}{|c|c|c|c|}
\hline Initial signal & Final signal & Phase shift [rad] & Attenuation [dB] \\
\hline BPSK & QPSK & $(2 \mathrm{n}+1) \pi / 2$ & 0 \\
\hline QPSK & 16QAM & $\mathrm{n} \pi / 2$ & $6 \mathrm{~dB}$ \\
\hline 16QAM & $256 \mathrm{QAM}$ & $\mathrm{n} \pi / 2$ & $6 \mathrm{~dB}$
\end{tabular}

The coherent superposition of the two electric fields in each DLI allows temporal compression of the information carried by the original signal by doubling its number of bits per symbol without spectral broadening. However, to fully take advantage of this compression function the information redundancy, caused by the presence of both the original symbol and its delayed replica, needs to be removed. In the proposed scheme this is achieved using a time-gating switch with a repetition rate that is two times the one of the DLI. In this way, while the format converted signal has the same information content as the original one, it occupies half of its space in time, which could be filled in with different information, i.e. a different packet in our specific case. As sketched in Fig.1 (a) this added packet needs to be time-interleaved and wavelength shifted to the same carrier. Note that this packet compression scheme based on signal recoding will necessitate the use of data pre-coding. Finally, it is shown in Fig.1 (b) how it is possible to scale the scheme to even higher compression factors, reaching more complex modulation formats to increase the number of bits per symbols. This is achieved by cascading multiple DLIs with appropriately adjusted delays. Once more, a single time-gating switch can be included at the end to remove the redundant information and the empty spaces can be filled in by different signals (packets), properly time-interleaved and wavelength shifted to a common carrier.

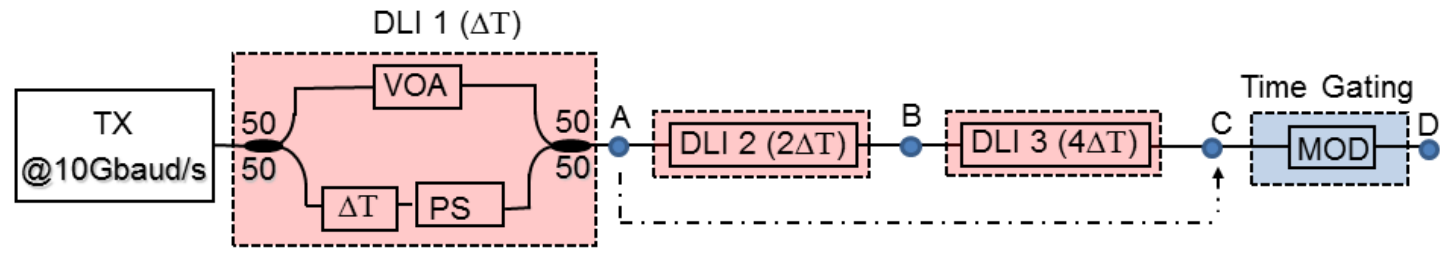

Fig. 2: Simulated set-up. TX: transmitter, VOA: variable optical attenuation, PS: phase shifter, MOD: amplitude modulator.

The simulated set-up of the packet compression scheme based on the passive coherent optical superposition scheme is shown in Fig.2. Two different systems were simulated. In the first one, a 10 Gbaud non-return-to-zero binary phase shift keying, BPSK, (or QPSK) signal was modulated onto a narrow-linewidth continuous wave (CW) laser at $193.1 \mathrm{THz}$ and launched into a first DLI to temporally multiplex the signal and achieve a QPSK (or 16 QAM) signal, point A in Fig.2. A 4096-bit time delay (409.6ns at 10Gbaud/s) was used in the DLI for both cases. The corresponding modulation format signal was then time gated with a synchronously driven amplitude modulator and characterised in terms of temporal waveforms and constellation diagrams. In the second one, a 10Gbit/s BPSK signal was launched into three cascaded DLIs with time delays equal to 1024, 2048 and 4096 bits respectively to simultaneously reach a 256QAM signal, compressing the original packet by a factor of 16 , point $\mathrm{C}$ in Fig.2. Once more, the new de-coded signal is then gated in time using a synchronously driven amplitude modulator and characterised. 


\section{RESULTS}

The numerical simulations were carried out using VPI Transmission Maker 8.0. For the initial results, the CW laser source was considered to have a null linewidth and no transmission was considered. Figure 3 shows the temporal waveforms and constellation diagrams at various points of the packet compression scheme (points A and D) when the input signals were BPSK (Fig.3 (a), and Fig.3 (b)) and QPSK (Fig.3 (c) and Fig.3 (d)). The original BPSK (QPSK) signal had an error vector magnitude (EVM) of $0.8 \%$ root mean square (rms) (1.4\% rms). After temporal multiplexing to QPSK (16QAM) and time gating the EVM became 0.9\%rms (1.5\% rms). It is worth pointing out that the temporal waveforms in Fig.3 (a) and Fig.3 (b) (as well as Fig.3 (c) and Fig. 3(d)) contain the same information, with Fig.3 (b) (Fig.3 (d)) occupying half of the time slot available, effectively compressing the original packet by a factor of 2. The zero level in the constellation diagram for the gated QPSK and 16QAM signals underlines the fact that the redundant signal was properly cleared and a second packet could occupy this empty time window to increase the overall SE.

By cascading three DLIs before the time gate, as shown in Fig.2, it is possible to directly go from a BPSK to a 256QAM signal (EVM of $1 \% \mathrm{rms}$ ), see Fig.4. In this case, due to the recoding of the signal, the same information can occupy 1/16 of the original time window, as shown in Fig.4 (b), reaching a compression factor of 16 .
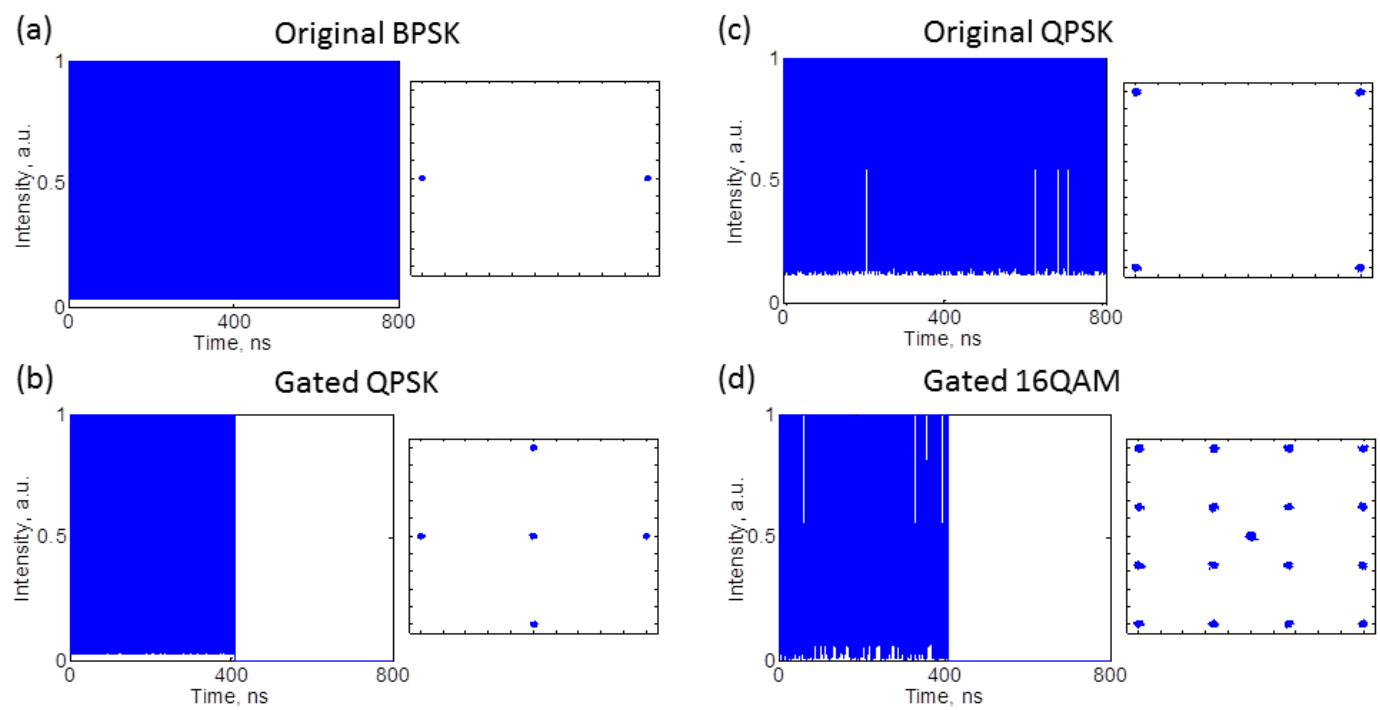

Fig. 3: Simulated temporal waveforms and constellation diagrams at the input/output of the packet compression scheme for BPSK (left) and QPSK (right) as input signals.
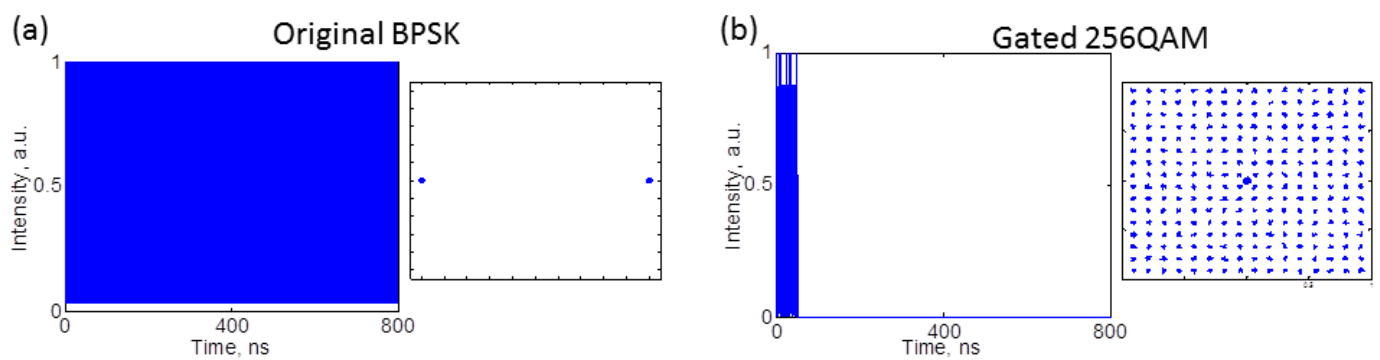

Fig. 4: Simulated temporal waveforms and constellation diagrams at the input (a) and output (b) of the packet compression scheme to achieve a compression factor of 16 with a BPSK as initial signal.

Finally, we assessed the scheme in terms of transmission distance before the format converter and initial laser linewidth. BPSK to QPSK format conversion at $10 \mathrm{Gbaud} / \mathrm{s}$ is considered for these studies. As the signal propagates through a transmission link it accumulates dispersion, broadening in time. Normally, this dispersion can be compensated for at the receiver, either optically, e.g using dispersion compensating fiber, or postreception, using electrical or digital filters. The above scheme however, is nonlinear in nature and therefore any accumulated dispersion prior to the device cannot be perfectly compensated for. Infact, we expect to observe a penalty in the presence of dispersion due to the loss of some signal information during the gating. As such, for ideal operation any dispersion should be compensated for prior to the receiver, at the gating input. We study this 
effect (impact of dispersion) by calculating the EVM of a signal after the device for various amounts of dispersion, which are only compensated at the receiver. Fig.5 (a), top, shows how the EVM values of the signal at the output of the scheme varies as a function of the transmission distance $\left(D_{S M F}=16 \mathrm{ps} / \mathrm{nm} / \mathrm{km}\right)$ for various values of the delay in the DLI. The impact depends on the length of the delay used in the DLI, relative to the extent of pulse broadening due to dispersion. For long delays (such as 4096 bits), while the pulses are broadened in time most of them still fit within the gating window, and therefore are affected minimally by the gating process. As the delay decreases, more and more of the pulses extend out of the gating window, hence an increased penalty. The bottom of Fig. 5(a) reports a table of maximum transmission distances for the discussed delays to achieve a $20 \%$ rms EVM. We have also investigated the impact of laser linewidth on the performance of the system. As would be expected, the longer the delay, the greater the required laser coherence length (as the bits must interfere coherently), as can be seen in Fig. 5 (b). As such a compromise may have to be made between dispersion tolerance and linewidth requirements, which respond in a complimentary manner to increasing delay.
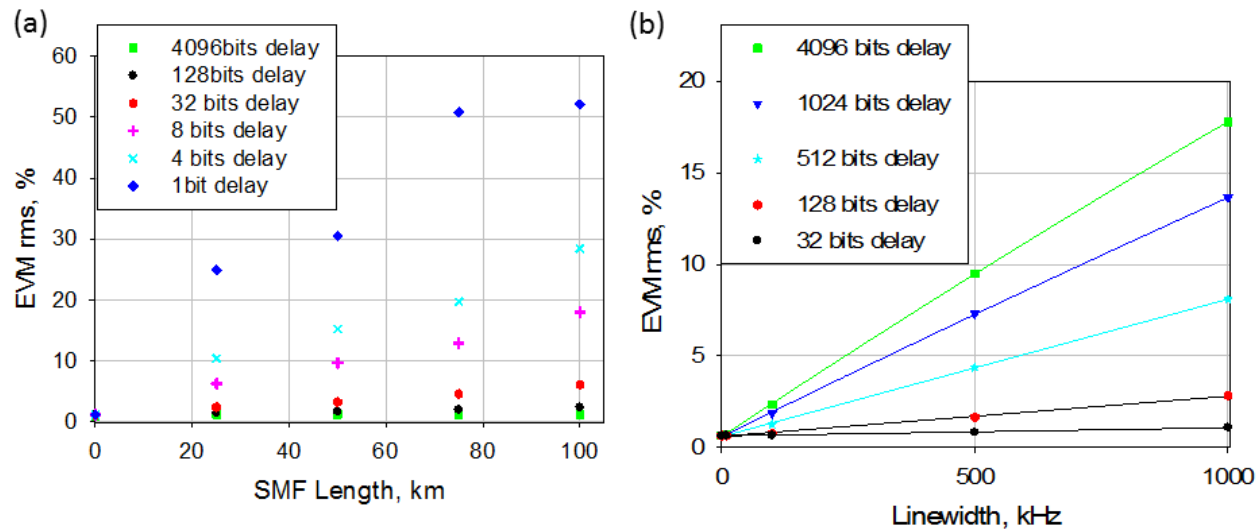

\begin{tabular}{|l|l|l|l|l|l|l|}
\hline Delay, bits & 1 & 4 & 8 & 32 & 128 & 4096 \\
\hline $\begin{array}{l}\text { Transmission } \\
\text { length, km }\end{array}$ & $\mathbf{2 5}$ & $\mathbf{7 5}$ & $\mathbf{1 0 0}$ & $\mathbf{6 0 0}$ & $\mathbf{3 0 0 0}$ & $\mathbf{1 0 5 0 0 0}$ \\
\hline
\end{tabular}

Fig. 5: a) Top: simulated EVM values versus transmission length prior th packet compression scheme for different DLI delays (switch time windows ). Bottom: Maximum reach for various delays to achieve EVM $\sim 20 \%$ rms. b) Simulated EVM values as a function of laser linewidth for different values of DLI delays.

\section{CONCLUSION}

In conclusion, we have presented and numerically demonstrated a packet compression scheme, in which passive coherent superposition of data bits was combined with time gating to eliminate redundant information and increase the number of bits per symbol. A variety of complex modulation formats can be processed and the packet compression factors can be easily scaled achieving higher order modulation formats. In the simulation, cascading three DLIs together, a compression factor of 16 has been shown. Dispersion tolerance and linewidth requirements of the scheme were also investigated. Experimental work will be carried out to validate the simulations.

\section{ACKNOWLEDGEMENTS}

Dr. F. Parmigiani gratefully acknowledges the support from the Royal Academy of Engineering/EPSRC through a University Research Fellowship. This work is supported by the EPSRC grant EP/I01196X: Transforming the Future Internet: The Photonics Hyperhighway.

\section{REFERENCES}

[1] A. H. Gnauck and P.J. Winzer, J. Lightwave Technol. 23 (1), 115-130 (2005).

[2] C. Xu, X. Liu, and X. Wei, IEEE J. Sel. Topics Quantum Electron. 10 (2), 281-293 (2004).

[3] C. Xu, et al., IEEE Photon. Technol. Lett. 15, 617-619 (2003).

[4] K. Mishina et al. Optics Express, 15 (13), 8444-8453 (2007).

[5] G. W. Lu et al. J. Lightwave Technol. 29 (16), 2460-2466 (2011).

[6] J.Kakande, et al., ECOC, PD 3.3 (2010).

[7] H. Kishikawa, et al., Photonics Conference (PHO), 513 - 514 (2011).

[8] M. Sköld et al., ECOC PD Th.13.B.3 (2011). 